shows the number and percentage of adenomas found in the left colon (up to and including the splenic flexure) and the right colon (proximal to the splenic flexure) in both the index and surveillance procedures.

Significantly more adenomas were identified within the right colon at the surveillance procedures than were in the index examinations $(\mathrm{p}=0.0001)$.

Conclusion This study suggests that more proximal adenomas are found during surveillance colonoscopies than on initial screening colonoscopies. As approximately $60 \%$ of the surveillance examinations were at one year it is likely that these lesions were missed at the initial examination. Greater care needs to be taken at initial colonoscopy to visualise the proximal bowel and clear it of neoplasia, particularly in those individuals with multiple polyps.

Disclosure of Interest None Declared.

\section{PWE-026 MULTIPLE POLYPS HAVE A HIGHER PREDICTIVE VALUE FOR POSITIVE FINDINGS ON SURVEILLANCE COLONOSCOPY THAN FEWER LARGE LESIONS}

A Kamora*, K Kandiah, G Smith, J Martin. Gastroenterology Department, Imperial College Healthcare NHS Trust, London, UK

\subsection{6/gutjnl-2014-307263.286}

Introduction Individuals with adenomatous polyps are at increased risk of developing further neoplasia and it is recommended that these individuals undergo colonoscopic surveillance at an interval dictated by the size of the adenoma and/or the number of adenomas removed. This study documents the findings at surveillance colonoscopies in individuals within the Bowel Cancer Screening Programme (BCSP).

Methods All patients undergoing surveillance colonoscopies within the BCSP at West London Bowel Cancer Screening Centre (WLBCS) between $1^{\text {st }}$ January 2009 and $1^{\text {st }}$ February 2013 were were stratified into four groups according to the findings at the index colonoscopy as follows:

Group A -3 or more adenomas with at least one $=10 \mathrm{~mm}$

Group B -5 or more small adenomas all $<10 \mathrm{~mm}$

Group $\mathrm{C}-1$ or 2 adenomas $=10 \mathrm{~mm}$

Group D -3 or 4 small adenomas all $<10 \mathrm{~mm}$

The percentage of adenomas found at surveillance colonoscopy in patients with few adenomas at index colonoscopy was compared to adenoma yield in patients with multiple adenomas at index colonoscopy using Fishers exact test.

Results 242 patients underwent colonoscopies within the study period and a total of 379 adenomas were found in 145 patients. 19 adenomas were greater than $10 \mathrm{~mm}(5.0 \%)$ and 3 adenomas showed high grade dysplasia $(0.8 \%)$. The percentage of patients with adenomas found within the groups A-D is shown in the table below.

Overall the percentage of patients with adenomas was $69.0 \%$ in those patients having their surveillance procedure at 1 year and $47.0 \%$ in those individuals having their colonoscopy at 3 years. For both surveillance intervals, the group with the largest number of adenomas found at the index procedure had a higher adenoma yield during surveillance. Overall, adenomas were found in $67.8 \%$ of patients with multiple adenomas found at the index colonoscopy (groups A, B, D) and in $43.6 \%$ of patients with 1 or 2 larger lesions (group $C)(p=0.0004)$.

Conclusion Adenoma detection at surveillance colonoscopy within the BCSP is high, at both the 1 year and the 3 year interval, suggesting that this is a valuable intervention in reducing future risk of colorectal cancer. The yield of high-risk lesions of a large size or with high-grade dysplasia, however, is low. The yield of adenoma detection is higher in individuals undergoing surveillance for high numbers of diminutive lesions compared to those individuals with 1 or 2 large adenomas. This finding may reflect the presence of a generalised colonic field effect in those individuals with multiple lesions, compared to a more local abnormality in those with small numbers of lesions.

Disclosure of Interest None Declared.

\section{PWE-027 THE REASONS FOR GENDER DIFFERENCES IN CAECAL INTUBATION RATES - ANALYSIS OF 8324 COLONOSCOPIES OVER 6 YEARS}

${ }^{1}$ AM Verma*, ${ }^{1}$ RE Smith, ${ }^{2} \mathrm{~N}$ McGrath, ${ }^{1}$ A Dixon, ${ }^{1}$ AP Chilton. ${ }^{1}$ Gastroenterology, Kettering General Hospital NHS Foundation Trust, Kettering, UK; ${ }^{2}$ Endoscopy, Kettering General Hospital NHS Foundation Trust, Kettering, UK

\subsection{6/gutjnl-2014-307263.287}

Introduction In 2012 we presented a poster to the Digestive Disorders Foundation Meeting, we analysed 5162 colonoscopies and noted a significant difference in caecal intubation rates (CIR) of male and female patients $(92.73 \%$ v $87.63 \%, \mathrm{p}<$ 0.0001, NNH 19.57). ${ }^{1}$

Gender differences in colonoscopy have been published previously in the $1990 \mathrm{~s}^{2,3}$ Several theories were mooted for this difference; such as female patients undergoing previous hysterectomy, ${ }^{2}$ and having longer colons. ${ }^{3}$ We have revisited this topic to identify causes of the difference relevant to modern colonoscopic practice.

Methods Data was analysed from 8324 colonoscopies at Kettering General Hospital 2008-13. Incomplete colonoscopies' reports were scrutinised to record the causes of failure.

Results Reason for failed colonoscopy (females v males, $\mathrm{p}$ value)

Poor bowel preparation (16.38 vs. $24.66 \%, 0.09)$, tight bend (6.21 vs. $0.91 \%,<0.03)$

Intolerance/pain (27.97 vs. $19.63 \%, 0.11)$, looping (18.36 vs. $18.72 \%)$

Obstructing lesion (8.19 vs. $15.53 \%, 0.06)$, previous surgery (5.37 vs. $0.46 \%,<0.03$ )

Diverticular disease $(9.32$ vs. $5.02 \%, 0.18)$, withdrew consent (5.93 vs. $2.28 \%, 0.14$ )

\begin{tabular}{llll} 
Abstract PWE-026 Table 1 & & & \\
\hline & & Surveillance colonoscopies performed $\mathbf{n}$ & Number with adenomas \\
Surveillance protocol & Group A & 121 & $80(66)$ \\
\hline 1 year surveillance & Group B & 21 & $18(86)$ \\
3 year surveillance & Group C & 78 & $34(44)$ \\
& Group D & 22 & $13(59)$ \\
& & 242 & $145(60)$ \\
\hline
\end{tabular}




\begin{tabular}{|c|c|c|c|c|c|}
\hline & Number of colonoscopies & Reached caecum/TI/anastomosis & Incomplete & CIR & $95 \% \mathrm{Cl}$ \\
\hline Female & 3886 & 3532 & 354 & $90.89 \%$ & 89.94-91.76 \\
\hline Male & 4438 & 4219 & 219 & $95.07 \%$ & $94.39-95.66$ \\
\hline Total & 8324 & 7751 & 573 & $93.12 \%$ & $92.55-93.64$ \\
\hline
\end{tabular}

Conclusion The data reveals significant differences in CIR between female and male patients $(90.89$ vs. $95.07 \%$, p < $0.0001, \mathrm{NNH} 24)$. Analysis of the reasons recorded for failure shows a strong trend in males for poor bowel preparation and obstructing lesion. In females, a strong trend was shown for pain/intolerance, diverticular disease and withdrawal of consent. Statistical significance was shown for previous (abdominal) surgery and tight bend. Looping is a common reason for failing colonoscopy with no gender difference.

This is an important observation that females are significantly less likely to have complete colonoscopy. Perhaps endoscopy units should outline the potential for missed lesions as a consequence when consenting female patients - in particular those with known diverticular disease or previous abdominal surgery. Other reasons of failure could also be addressed e.g. higher doses of analgesia for females as required.

\section{REFERENCES}

1 VermaAM, McGrath N, Dixon A, Chilton AP. Gender differences: analysis of 5162 colonoscopies over 4 years reveals higher caecal intubation rates in male patients. Gut 2012;61:Suppl 2 A150-A151

2 Church JM. Complete colonoscopy: How often? And if not, why not? Am J Gastroenterol 1994;89:556-60

3 Saunders BP, Fukumoto $M$, Halligan $S$, et al. Why is colonoscopy more difficult in women? Gastrointestinal Endoscopy 1996:43:124-6.

Disclosure of Interest None Declared.

\section{PWE-028 PATIENT COMFORT AND SEDATION AND ANALGESIC PRACTICES DURING COLONOSCOPY IN THE ENGLISH BOWEL CANCER SCREENING PROGRAMME}

A Ball*, SA Riley. Gastroenterology, Sheffield Teaching Hospitals, Sheffield, UK

\subsection{6/gutjnl-2014-307263.288}

Introduction Colonoscopy frequently causes discomfort and a range of medications are used to improve the patient experience. The relationship between medication use and patient comfort, however, is complex and subject to a number of potential biases. We sought to describe the relationship between patient comfort and medication use within the English Bowel Cancer Screening Programme (BCSP).

Methods Procedural information for colonoscopy examinations performed within the English BCSP is prospectively entered into a national database. Comfort is independently rated by a specialist screening practitioner (SSP) using the Modified Gloucester Comfort Scale (no, minimal, mild, moderate and severe). We studied significant patient discomfort (moderate or severe) and medication usage for colonoscopists performing over 100 examinations between January 2010 and December 2012. Comparisons were made using the $\chi 2$ test and correlations were analysed using Spearman rank correlation coefficient.

Results During the period of the study 113,316 examinations were performed by 290 endoscopists. Significant discomfort occurred during $8.9 \%$ of colonoscopy examinations but there was variation between individual colonoscopists (median 8.1\%,
IQR 5.0-12.6\%, range 0.8-23.9\%). Significant discomfort was more common in females (12.7 vs. $6.1 \%$, odds ratio (OR) 2.24), patients with diverticulosis $(11.8 \%$ vs. $8.7 \%$, OR 1.34$)$, incomplete examinations (37.3 vs. $7.9 \%$, OR 6.8), inadequate bowel preparation (13.5 vs. 9.6\%, OR 1.4) and screening rather than surveillance colonoscopies (9.1 vs. 7.4\%, OR 1.24).

Midazolam was administered during $87.8 \%$ and opiate analgesia during $87.3 \%$ of procedures. There was wide variation between colonoscopists in the proportion of examinations in which midazolam (median use 95.1\%, IQR 81.8-97.8\%, range 4.1\% $>100 \%$ ) and opiate analgesia (median use 97.3, IQR 85.099.2\%, range 5.6-100\%) were used. Reversal agents were rarely used (8 in 10,000). Entonox was administered during 7.5\% of examinations but most who administered it did so in a minority of their procedures (median use $0.7 \%$, IQR $0-8.2 \%$, range 0 98.9\%). $4.7 \%$ of patients underwent medication-free colonoscopy. General anaesthesia was rarely used $(0.5 \%)$.

There were no significant correlations between the amount or proportion of medication used by colonoscopists and the comfort of their patients.

Conclusion Most colonoscopy examinations were performed without causing significant discomfort. Although most colonoscopists used intravenous medication those who used less medication were no more likely to cause significant discomfort. Appropriate use of medication to achieve comfortable procedures while minimising risk and inconvenience remains an important focus for future research.

Disclosure of Interest None Declared.

\section{PWE-029 POSITION CHANGES AMONG ENGLISH BCSP COLONOSCOPISTS: A SURVEY OF PRACTICES}

A Ball ${ }^{*}$, J Campbell, SA Riley. Gastroenterology, Sheffield Teaching Hospitals, Sheffield, UK

\subsection{6/gutjnl-2014-307263.289}

Introduction Studies suggest that modifying a patient's position during colonoscope withdrawal may improve luminal distension and polyp detection. It is unclear whether this practice is widely adopted by endoscopists.

Methods Colonoscopists within the English Bowel Cancer Screening Programme (BCSP) were invited to participate in a web-based survey assessing the use of position change during colonoscope withdrawal. Free text responses were assessed using thematic analysis.

Results The survey was completed by 204/298 (68\%) of English BCSP colonoscopists. $64.7 \%$ of respondents indicated that they almost always change a patient's position, $16.7 \%$ usually, $13.7 \%$ sometimes, $3.4 \%$ occasionally and $1.5 \%$ rarely do so.

$77 \%$ of those who almost always or usually changed a patient's position did so as part of their routine, but $75.3 \%$ were less likely to change position in those with poor mobility and $75.3 \%$ would not change position if luminal distension was adequate. $93 \%$ of these respondents most often positioned 